STUDIA PRAWNO-EKONOMICZNE, T. CXI, 2019

PL ISSN 0081-6841; e-ISSN 2450-8179 $\quad$ s. 11-30

https://doi.org/10.26485/SPE/2019/111/1

\title{
Farida ARHAB-GIRARDIN*
}

iD https://orcid.org/0000-0003-3830-140X

\section{QUELLES MESURES DE PROTECTION DES PERSONNES ATTEINTES DE LA MALADIE D'ALZHEIMER? WHAT PROTECTIVE MEASURES FOR PEOPLE WITH ALZHEIMER'S DISEASE?}

\begin{abstract}
(Summary)
The number of people suffering from Alzheimer's disease or other neurodegenerative diseases is growing up with the ageing of the population. However, they raise new questions because the alteration of mental faculties is gradual and definitive in the present state of knowledge. Many patients, although suffering from cognitive difficulties, are still able to participate in decision-making. The objective of the study is to measure the adequacy of the various existing protective measures for this type of disease. It shows that modern measures of conventional or weakly judicial protection are still perfectible, and the traditional judicial measures have the advantage of graduating the protection adapted to the evolution of the state of health of the person are suffering from Alzheimer's disease but it proves to be ineffective.
\end{abstract}

Keywords: mandate for future protection; judicial protection measures; conventional protection; Alzheimer's disease

Le vieillissement de la population s'accompagne d'une augmentation des maladies liées au grand âge dont la maladie d'Alzheimer ${ }^{1}$. Celle-ci est définie comme «une maladie dégénérative progressive et irréversible du système nerveux central, responsable d'un déclin cognitif, de troubles de la mémoire

* Maître de Conférences de droit privé à l'Université de Tours, France; e-mail: farida.arhab-girardin@univ-tours.fr

1 En France, le nombre estimé de personnes atteintes de la maladie d'Alzheimer et d'autres démences était compris entre 750000 et 1000000 en 2010, avec des projections pour 2030 atteignant 1,29 à 1,40 million de patients. F. Semah, W. Deberdt, M. Ceccaldi, De la maladie d'Alzheimer: apport de l'imagerie au florbétapir et autres radiopharmaceutiques de la plaque amyloïde, Médecine et droit, vol. 40, p. 364 et s. 
et de troubles comportementaux, qui interfèrent avec les activités de la vie quotidienne et génèrent un état de dépendance croissante $»^{2}$. Les troubles cognitifs s'installent progressivement jusqu'à la démence. Une période de déclin cognitif et mnésique précède le stade avéré de démence 3 .

La personne atteinte de la maladie d'Alzheimer devient, au fur et à mesure de son évolution, un majeur vulnérable qui a besoin de protection. Elle ne perd cependant pas immédiatement son autonomie. Il n'en reste pas moins que dès les premiers stades de la maladie, la protection peut s'avérer nécessaire en préservant autant qu'il est possible son bien-être ${ }^{4}$. Il a toutefois été constaté que de nombreuses personnes atteintes de la maladie ne font pas l'objet de mesures de protection immédiates ${ }^{5}$. Ce phénomène peut en partie s'expliquer par le fait qu'une personne vulnérable ne doit être placée sous un régime de protection juridique que si aucun autre mécanisme plus léger ne peut être mis en œuvre. Lorsque ces mécanismes sont insuffisants ou défaillants, le déclenchement d'une mesure juridique destinée à la protéger s'impose. A cet égard, les personnes atteintes de la maladie d'Alzheimer ne constituent pas une catégorie juridique à part, elles sont soumises aux règles du droit commun de la protection des majeurs.

En ce domaine, l'une des problématiques soulevée est d'assurer la nécessaire protection juridique des personnes vulnérables tout en préservant leurs droits fondamentaux. La loi du 5 mars 2007 portant réforme de la protection juridique des majeurs entend réaliser cet objectif ${ }^{6}$, «protéger sans diminuer ${ }^{7} »$. La loi n ${ }^{\circ} 2015$ -1776 du 28 décembre 2015 relative à l'adaptation de la société au vieillissement le réitère. Le respect de la personne protégée doit, en conséquence, toujours gouverner la mesure de protection laquelle s'étend depuis 2007 à la protection de la personne et non plus seulement à celle du patrimoine. Cette double protection s'impose comme une véritable nécessité juridique mais également éthique ${ }^{8}$ comme l'exprime l'article 415 du code civil: «Les personnes majeures reçoivent la protection de leur personne et de leurs biens que leur état ou leur situation

Ibidem.

Ibidem.

4 A. Caron-Déglise, Protéger pour construire les capacités des personnes malades, in: Alzheimer, éthique et société, sous dir. de F. Gzil, E. Hirsch. ERES 2012, p. 303 et s.

5 B. Bévière-Boyer, Quel consentement pour les patients atteints par la maladie d'Alzheimer lors des recherches?, in: Consentement et santé, Dalloz, thèmes et commentaires, p. 333 et s.

Loi n 2007-308, JO 7 mars 2007, p. 4325, entrée en vigueur le $1^{\text {er }}$ janvier 2009.

7 T. Fossier, L'objectif de la réforme du droit des incapacités, protéger sans jamais diminuer Defrénois 2005, n³8076, p. 3.

8 R. Gil, Comment informer les patients atteints de troubles cognitifs?, RGDM 2016, n 59 , p. 38 et s. 
rend nécessaire [...]. Cette protection est instaurée et assurée dans le respect des libertés individuelles, des droits fondamentaux et de la dignité de la personne. Elle a pour finalité l'intérêt de la personne protégée. Elle favorise, dans la mesure du possible, l'autonomie de celle-ci. Elle est un devoir des familles et de la collectivité publique». Un équilibre entre le respect des libertés individuelles, la dignité et les besoins de protection doit donc être atteint ${ }^{9}$.

La protection des personnes atteintes de la maladie d'Alzheimer ou d'autres maladies neurodégénératives représente un véritable enjeu de société. Il existe actuellement une variété de mesures de protection susceptibles d'être mises en œuvre avec différentes combinaisons possibles en confiant un rôle prioritaire à la famille ${ }^{10}$. Il a été renforcé en $2015^{11}$ avec l'introduction d'une nouvelle mesure dans le paysage juridique français, l'habilitation familiale. La famille représente un soutien important à la personne vulnérable, mais les considérations économiques ne sont pas non plus étrangères à ce principe de priorité. La famille demeure malgré tout au cœur des mesures de protection de la personne qu'elles soient ou non judiciaires non seulement par la confiance qu'elle inspire ${ }^{12}$ mais également parce qu'elle apparaît comme une cellule fondamentale dans la vie des personnes vulnérables ${ }^{13}$. C'est seulement en cas de défaillance de celle-ci que les pouvoirs publics y suppléent en désignant un mandataire à la protection juridique des majeures.

Toutes ces mesures ne peuvent être ordonnées qu'en cas de nécessité (article 428 c.c.), si la personne est dans l'impossibilité de pourvoir seule à ses intérêts en raison d'une altération médicalement constatée de ses facultés personnelles, physiques ou mentales, empêchant l'expression de la volonté (article 425 c.c). En outre, elles ne peuvent être que subsidiaires et proportionnées. Elles doivent dans tous les cas concilier le respect de l'autonomie avec l'impératif de protection ${ }^{14}$.

La maladie d'Alzheimer soulève cependant des questions inédites dans la mesure où l'altération des facultés mentales est progressive et définitive en l'état actuel des connaissances. De nombreux malades, bien que souffrant de

9 Protéger les majeurs vulnérables. Quelle place pour les familles?, sous dir. de K. Leufeuvre, S. Moisdon-Chataigner, p. 193, presses de l'EHESP 2015.

10 K. Lefeuvre, La place de la famille dans la loi du 5 mars 2007 portant réforme de la protection juridique des majeurs, in: Protéger les majeurs vulnérables..., p. 31 et s.

11 Ordonnance $\mathrm{n}^{\circ} 2015-1288$ du 15 octobre 2015 portant simplification et modernisation du droit de la famille.

12 K. Lefeuvre, op. cit.

13 B. Feuillet, De la légicimité de la famille à l'intérêt du majeur protégé, in: Protéger les majeurs vulnérables..., p. 23.

14 Avis sur le consentement des personnes vulnérables, 16 avril 2015, Commission nationale consultative des droits de l'homme, JORF n 015810 juil. 2015. 
difficultés cognitives, sont encore capables de participer à la prise de décision ${ }^{15}$. Les moments de lucidité alternent également avec les troubles cognitifs.

Aussi, se pose la question de l'adéquation des différentes mesures de protection existantes à ce type de maladie. Dans le cadre de la législation sur les majeurs protégés, les unes sont prioritaires. Il en est ainsi de deux mesures récentes: d'une part, le mandat de protection future ${ }^{16}$ parce qu'il permet d'anticiper ses volontés et de les respecter ensuite lorsque la personne sera devenue inapte à pourvoir seule à ses intérêts; d'autre part, la nouvelle habilitation familiale qui est une mesure judiciaire simplifiée. Ces deux mesures modernes, l'une conventionnelle, l'autre faiblement judiciarisée ne sont pas encore les plus répandues et sont perfectibles (I). A titre subsidiaire, les mesures judiciaires classiques peuvent être mises en œuvre. Elles présentent l'avantage d'opérer une gradation de la protection adaptée à l'évolution de l'état de santé de la personne atteinte de la maladie d'Alzheimer mais cette gradation se révèle ineffective ${ }^{17}$ (II).

\section{La perfectibilité des mesures modernes de protection conventionnelles ou faiblement judiciarisées}

Le mandat de protection future est un instrument privilégié qui permet d'anticiper conventionnellement la protection de la personne (A). Il prime sur les dispositifs judiciaires ainsi que sur l'habilitation familiale laquelle constitue une nouvelle mesure de protection juridique simplifiée (B).

\section{A. Le mandat de protection future: une anticipation conventionnelle de la protection}

Le mandat de protection future, instrument de protection déjudiciarisé au service de la volonté de la personne (1), marque une avancée majeure dans le respect des droits de la personne vulnérable même s'il présente des imperfections dont certaines sont spécifiques à la situation des personnes atteintes de la maladie d'Alzheimer (2).

15 V. Depadt-Sebag, L'EREMA à la recherche d'un statut des personnes atteintes de la maladie d'Alzheimer, D. 2011. 2720. V. Lefebvre-des-Noettes, Le point de vue d'un médecin: le choix de Sophie entre conflit de loyauté et conflit d'intérêt, in: Protéger les majeurs vulnérables..., p. 207 et s. spéc. p. 210.

16 Civ. $1^{\text {re }}, 4$ janv. 2017, D. 2017.191, note D. Noguéro.

17 Rapport de la Cour des comptes, La protection juridique des majeurs: une réforme ambitieuse, une mise en œuvre défaillante, septembre 2016, http://www.ccomptes.fr/Accueil/Publications 


\section{Un instrument au service de la volonté de la personne}

Une anticipation de la volonté de la personne. Les droits des personnes particulièrement vulnérables à décider pour elles-mêmes sont loin d'être toujours respectés ${ }^{18}$. Le mandat de protection future peut y remédier parce qu'il apparaît comme l'instrument le plus respectueux de la volonté de la personne. Il permet d'associer la personne à sa propre protection ${ }^{19}$. Dès les premiers signes de la maladie d'Alzheimer voire avant son apparition éventuelle, il est possible d'y recourir ${ }^{20}$. Originaire du Québec et introduite en droit français par la loi du 5 mars 2007, cette institution permet à toute personne d'organiser de façon anticipée la protection de ses biens et de sa personne pour le jour où ses facultés corporelles et mentales seraient altérées l'empêchant de pourvoir seule à ses intérêts ${ }^{21}$.

C'est un mandat purement conventionnel, obligatoirement écrit, notarié ou sous seing privé soit contresigné par un avocat soit rédigé selon un modèle type réglementaire, par lequel une personne majeure ne faisant pas l'objet d'une mesure de tutelle ou d'une habilitation familiale (ou mineure émancipée) en pleine possession de ses moyens désigne, en dehors de toute intervention du juge, une ou plusieurs personnes chargée(s) de la représenter en cas d'altération de ses facultés mentales ou physique dans l'avenir. La mesure ne prend ensuite effet qu'en cas de besoin, sous le contrôle de la justice. Le mandat de protection future est prioritaire sur les mesures judiciaires de protection ${ }^{22}$.

La souplesse du mandat. Le recours au mandat de protection future peut permettre de mieux préserver les souhaits de la personne atteinte de la maladie et son autonomie avant que sa volonté ne soit altérée et évite le recours à une mesure judiciaire de protection ${ }^{23}$. Il est donc utile de le rédiger avant la progression de la maladie, lorsqu'une personne, se sachant atteinte de la maladie, a encore la capacité de consentir. Il est cependant préférable d'obtenir les conseils avisés de l'entourage voire d'un notaire tenu d'un devoir d'information et de conseil

18 Avis sur le consentement des personnes vulnérables, préc., Rapport du Défenseur des droits, Protection juridique des majeurs vulnérables, septembre 2016, http://www.defenseurdesdroits.fr/

19 L. Aynès et $\mathbf{P h}$. Mallaurie, Droit des personnes. La protection des mineurs et des majeurs, $8^{\text {ème }}$ éd. par Ph. Malaurie, 2016/737, p. 345.

20 Art. 477 et s. c.c.

21 Art. 477 c.c.

22 Principe rappelé par la Cour de cassation, Civ. $1^{\text {re }}, 4$ janv. 2017, préc.

23 Ch. Glasson, Droit et personnes âgées: Mandat de protection future: quelles dispositions peut-on prendre pour ses vieux jours?, NPG Neurologie - Psychiatrie - Gériatrie 2011-11(62), p. 55. 
ou de toute autre personne compétente. Le mandat notarié est celui qui réalise une conciliation entre la liberté individuelle du majeur et la sécurité juridique ${ }^{24}$. Cependant, son coût peut constituer un frein à son utilisation.

Le mandat de protection future est un instrument assez souple. A la différence des mesures judiciaires de protection, la personne désigne librement un ou plusieurs mandataires, personne physique (un membre de la famille, un proche, ou un tiers) ou même une personne morale mais uniquement celle inscrite sur la liste des mandataires judiciaires à la protection des majeurs afin d'éviter par exemple toute pression sectaire. En cas de conflit familial, le mandat peut imposer un tiers ou un proche parent. L'acceptation du mandataire est requise.

Pouvoirs du mandataire. Dès lors que la personne est dotée de ses facultés, elle est libre de délimiter les pouvoirs du mandataire chargé de la représenter. Elle peut limiter sa mission à la protection de son patrimoine ou à celle de sa personne, à l'image des mesures judiciaires de protection. Si elle confie au mandataire la protection de sa personne, le mandat peut s'appliquer à la décision médicale la concernant. Le mandant peut aussi prendre la précaution de donner des directives générales quant au choix de son lieu de vie, de ses relations, de ses visites, sa santé 25 , autant d'éléments importants lorsque les facultés dégénèrent. Il est aussi possible de répartir les compétences en désignant par exemple un mandataire chargé de la gestion du patrimoine tel un professionnel de confiance et un autre pour la prise en charge de la personne tel un proche. Le mandat notarié confère cependant des pouvoirs plus importants sur les biens que le mandat sous seing privé ${ }^{26}$. Il s'exerce en principe à titre gratuit mais il est rémunéré lorsque le mandataire est un professionnel.

Le mandat de protection future peut apparaître comme un instrument indispensable à « la concrétisation d'un pacte de prise en charge de la personne vulnérable qu'il faut protéger $\gg^{27}$. Il est recommandé de le réviser régulièrement pour l'adapter à l'évolution de sa situation patrimoniale et familiale tant que la personne a encore toutes ses facultés et avant son activation.

24 Rapport du Défenseur des droits sur la protection juridique du majeur vulnérable, préc.

25 V.F. Sauvage, Répertoire de droit civil, Mandat de protection future, $\mathrm{n}^{\circ} 30$. J. Klein, Le mandat de protection future ou la protection juridique conventionnelle, Dr. fam. 2007/21.

26 Le mandat notarié confère au mandataire la possibilité d'accomplir tous les actes patrimoniaux qu'un tuteur peut accomplir seul ou avec une autorisation du juge des tutelles (art. 490 c.civ.). L'autorisation du juge des tutelles est requise pour les actes de disposition à titre gratuit. Le mandat sous seing privé ne confère des pouvoirs que sur les actes conservatoires ou de gestion courante.

27 Avis sur le consentement des personnes vulnérables, préc. 
Afin d'être portée à la connaissance de toute personne intéressée, il doit désormais faire l'objet d'une publication sur un registre spécial ${ }^{28}$.

\section{Les imperfections du mandat de protection future}

Imperfections communes à tout mandat de protection future. On peut regretter que le contrôle judicaire ne soit pas toujours suffisant. Le juge, garant des libertés, n'intervient ni dans la conclusion, ni dans sa mise en œuvre ou son exécution, si ce n'est en cas de difficulté ou pour certains actes. Et pour cause, il s'agit d'un mandat conventionnel. De plus, celui-ci n'entraîne aucune incapacité et donc aucune protection particulière. Le mandant peut donc continuer à accomplir des actes. Or, il est vulnérable et s'expose aux sollicitations mal intentionnées. L'homologation du mandat ${ }^{29}$ par le juge pourrait bien être utile afin de vérifier l'absence de toute pression ou d'éviter son utilisation dans le but de contourner les mesures judiciaires et cela dès son élaboration ${ }^{30}$.

Le juge n'est cependant pas totalement absent. Il peut intervenir dans différentes circonstances. D'abord, lorsque le mandat de protection future est trop limité pour protéger suffisamment les intérêts personnels ou patrimoniaux de la personne, le juge prononcera une mesure de protection juridique «complémentaire» qui peut parfaitement être confiée au mandataire de protection future. Ensuite, le mandat peut faire l'objet de contestations, le juge saisi par la personne protégée elle-même, un membre de la famille ou un tiers tel un banquier ou un établissement d'hébergement pour personnes âgées dépendantes, peut y mettre fin et, si l'état de la personne l'impose, il prononce une mesure de protection judiciaire. Le rôle du juge est de préserver les intérêts de la personne protégée. La célèbre affaire Bétencourt en est une illustration. Le juge y a finalement mis fin en raison de graves dysfonctionnements constatés dans la gestion et a prononcé une mesure de tutelle confiée à la famille ${ }^{31}$.

Le mandat de protection future fonctionne mal dans une famille où les membres ne s'entendent pas. En pratique, il est préconisé de conjuguer un

28 Art. 477-1 c.c. issu de l'ordonnance 2015-1776 du 28 décembre 2015.

29 Rapport du Défenseur des droits sur la protection juridique des majeurs vulnérables, spéc. p. 22.

30 D. Noguéro, note sous Civ. $1^{\text {re }}, 4$ janv. 2017, préc.; Civ. $1^{\text {re }}, 12$ janv. 2011, D. 2011. 1204, note D. Noguéro; AJ fam. 2011. 110, obs. T. Verheyde; RTD civ. 2011. 323, obs. J. Hauser; JCP N 2011. 1115, note D. Boulanger; JCP 2011. 416, note N. Peterka; Dr. fam. 2011, n 42 , note I. Maria; LPA 2011, n 34, p. 10, note L. Gatti; Defrénois 2011, art. 39224, p. 690, note J. Massip; RJPF avr. 2011, p. 11, note A. Caron-Déglise.

31 L. Pécaut-Rivolier, Protéger un majeur vulnérable, Delmas express 2012, p. 44 et s. Sur cette affaire, cf. Ph. Malaurie, op. cit., p. 317, note 17. 
mandat de protection future et la désignation pour l'avenir du curateur ou du tuteur, dans un acte unilatéral, manuscrit, daté et signé. Ainsi, pour le cas où le juge des tutelles mettrait fin au mandat de protection future, il serait tenu par cette désignation anticipée du protecteur.

Imperfections spécifiques en présence d'une personne atteinte de la maladie d'Alzheimer. L'inconvénient majeur du mandat est qu'il ne peut être modulé en fonction de la progression de la maladie ${ }^{32}$. Il ne répond donc pas forcément aux différents degrés de son évolution. En effet, au début de la maladie, une simple assistance doit pouvoir suffire. Or, le mandat confère un pouvoir de représentation alors qu'une mesure de curatelle simple ou renforcée est préférable à ce stade ${ }^{33}$.

Enfin, il ne prend effet que lorsqu'une expertise médicale ${ }^{34}$ aura permis au greffier en chef du TGI de constater que le mandant ne peut plus pourvoir seul à ses intérêts pour l'une des causes prévues à l'article 425 du code civil, notamment en raison d'une altération médicalement constatée de ses facultés mentales de nature à empêcher l'expression de sa volonté. Le mandataire est accompagné du mandant sauf s'il est établi par certificat médical que sa présence au tribunal est incompatible avec son état de santé ${ }^{35}$. Une difficulté importante se pose cependant en pratique lorsque le mandant, dans le déni de la maladie, refuse de se soumettre à l'examen médical. Or, sans examen médical, le mandat de protection future ne pourra pas être mis en œuvre.

Il apparaît enfin que cette institution est peu utilisée car peu connue. Sa promotion auprès des familles et des proches s'avère donc indispensable ${ }^{36}$.

A défaut de mandat de protection future, une nouvelle mesure prioritaire peut être mise en œuvre: l'habilitation familiale.

\section{B. L'habilitation familiale: une nouvelle mesure de protection judiciaire simplifiée}

La solidarité familiale est au cœur de ce nouveau mandat judiciaire allégé (1), des améliorations sont cependant nécessaires (2).

32 D. Noguéro, Interrogations au sujet du mandat de protection future, Defrénois 2009/1133.

33 V. par ex. G. Raoul-Cormeil, Le mandat de protection future, un contrat pour préserver les biens professionnels ou l'intérêt de la famille, LPA 2014.

34 Le mandataire doit demander à un médecin inscrit sur la liste dressée par le Procureur de la République une attestation médicale.

35 Art. 1258-1 c. pr. civ.

36 L'avis de la CNCDH sur l'effectivité des droits des personnes âgées du 27 juin 2013 recommande de développer les actions d'information sur les dispositifs permettant d'organiser à l'avance la protection des personnes vulnérables et le respect de leurs volontés. 


\section{Un mandat judiciaire allégé reposant sur la solidarité familiale}

L'habilitation familiale, nouvelle mesure de protection juridique simplifiée entrée en vigueur le $1^{\text {er }}$ janvier $2016^{37}$, limite l'intervention du juge et évite le prononcé d'une tutelle. Elle permet aux proches d'une personne qui est hors d'état de manifester sa volonté de faire habiliter l'un d'entre eux par un juge pour la représenter dans la gestion de ses biens ou protéger sa personne ${ }^{38}$, en échappant au formalisme des mesures judiciaires ${ }^{39}$.

Elle est subsidiaire par rapport aux règles du droit commun de la représentation et au mandat de protection future ${ }^{40}$.

C'est une mesure hybride ${ }^{41}$. Elle se situe entre une mesure judiciaire de protection et un mode de représentation conventionnel ${ }^{42}$. Elle est ordonnée par le juge mais emprunte, dans le même temps, certaines règles du mandat de protection future.

C'est d'abord une mesure judiciaire puisque c'est le juge des tutelles qui, lorsqu'une personne est hors d'état de manifester sa volonté pour une des causes prévues par l'article 425 du Code civil, habilite une personne choisie parmi les proches afin de la représenter ou passer des actes en son nom dans le but d'assurer la sauvegarde de ses intérêts. Le rôle du juge demeure cependant limité.

Comme pour toute mesure de protection, elle ne peut être ordonnée par le juge qu'en cas de nécessitét ${ }^{4}$. Or, au fur et à mesure de l'évolution de la maladie d'Alzheimer, cette nécessité va s'imposer. L'habilitation familiale ne semble, cependant, pouvoir concerner que le patient en stade évolué de la maladie. Des éclaircissements seront sans doute apportés par la pratique judiciaire ${ }^{44}$ car l'altération de ses facultés mentales peut la mettre, ponctuellement ou de façon permanente, hors d'état de manifester sa volonté.

37 Ordonnance $\mathrm{n}^{\mathrm{0}} 2015-1288 \mathrm{du} 15$ octobre 2015 portant simplification et modernisation du droit de la famille. Décret nº 2016-185 du 23 février 2016.

38 G. Raoul-Cormeil, Les personnes protégées et les dispositifs d'anticipation sur la fin de vie médicalisée, Dr. fam., oct. 2016/10, dossier 35.

39 Rapp. au Président de la République, JO 16 oct. 2015, p. 19301.

40 Art. 494-2 c.c.

${ }^{41}$ G. Raoul-Cormeil, L'habilitation familiale: une tutelle adoucie, en la forme et au fond, D. $2015,2335$.

42 I. Maria, L'habilitation familiale, une nouvelle mesure de protection qui doit faire ses preuves, Dr. fam. 2016/11, dossier 5.

43 Article 494-2 c.civ.

44 T. Verheyde, Capacité ou non du majeur à protéger à exprimer sa volonté: des incohérences, dossier «Majeurs protégés: les nouveautés», AJ fam. 2016.236. 
L'habilitation familiale est ensuite une mesure de représentation qui emprunte au mandat de protection future. Le majeur conserve l'exercice de ses droits, autres que ceux dont l'exercice a été confié à la personne habilitée ${ }^{45}$. Elle entraîne une incapacité contractuelle ${ }^{46}$. L'habilitation peut être simple ou spéciale c'est-à-dire porter sur un ou plusieurs actes d'administration ou de disposition, et un ou plusieurs actes relatifs à la personne. Toutefois, si l'intérêt de la personne à protéger l'implique, le juge peut délivrer une habilitation générale. Les pouvoirs de l'habilité sont alors plus larges que ceux du tuteur puisqu'il peut passer seul tous les actes (patrimoniaux et personnels) ou tous les actes patrimoniaux ou tous les actes personnels ${ }^{47}$ sans avoir à distinguer les actes d'administration des actes de disposition. En cela, cette mesure se rapproche du mandat de protection future conclu sous forme notarié. L'ouverture d'un compte bancaire au nom du majeur protégé ou sa clôture est aussi dispensée d'une autorisation du juge. De plus, «la personne protégée choisit le lieu de sa résidence» ${ }^{48}$, tant que cela est possible.

Des limites sont cependant posées: la personne habilitée ne peut accomplir un acte de disposition à titre gratuit (donation par exemple) qu'avec l'autorisation du juge des tutelles ${ }^{49}$. De plus, elle ne peut accomplir un acte pour lequel elle serait en opposition d'intérêts avec la personne protégée ${ }^{50}$. Toutefois, à titre exceptionnel et lorsque l'intérêt de celle-ci l'impose, le juge peut autoriser la personne habilitée à accomplir cet acte.

Seule l'habilitation générale doit faire l'objet d'une publication obligatoire en marge de l'acte de naissance, et non l'habilitation spéciale ce qui est critiquable.

Comme pour les autres mesures de protection, l'altération doit être constatée par un certificat médical circonstancié. L'intéressé doit être entendu ou appelé. Sous certaines conditions, le juge peut «décider qu'il n’y a pas lieu de procéder à son audition si elle est de nature à porter atteinte à sa santé ou si la personne est hors d'état de s'exprimer ${ }^{51}$. Cependant, se dispenser d'une audition du majeur porte atteinte à ses droits fondamentaux.

\footnotetext{
Art. 494-8 c.c.

G. Raoul-Cormeil, L'habilitation familiale: une tutelle adoucie, en la forme et au fond, préc. Art. 494-6, al. 5, c.c.

Art. $459-2$ c.c.

Art. 494-6, al. 4, c.c.

Art. 494-6 c.c.

Art. 494-4, al. 1er c.c.
} 
Il s'agit, enfin, d'un mandat judiciaire ${ }^{52}$ dont l'objectif prioritaire est de renforcer davantage la solidarité familiale, en favorisant l'investissement régulier des «proches» d'une personne vulnérable ${ }^{53}$. La famille est donc placée au premier rang pour protéger le majeur vulnérable. L'accent est mis sur l'évitement du formalisme de la tutelle ou curatelle afin de séduire la famille souvent réfractaire aux mesures judiciaires. Cette mission est exercée à titre gratuit.

Une telle mesure suppose évidemment une certaine harmonie familiale ${ }^{54}$. Elle ne saurait être mise en œuvre en cas de conflits. Dans le souci d'instaurer la mesure dans un climat apaisé, le juge doit s'assurer de l'adhésion ou de l'absence d'opposition «légitime» des proches, à la fois à la mesure d'habilitation qu'au choix de la personne habilitée ${ }^{55}$. L'existence de conflits intrafamiliaux, l'éloignement et l'absence de la famille ou son souhait de ne pas exercer la mesure expliquent souvent que les autres mesures judicaires de protection soient confiées à un mandataire professionnel ${ }^{56}$. Il en sera sans doute de même de l'habilitation familiale.

\section{Les améliorations nécessaires de l'habilitation familiale}

L'habilitation familiale est restrictive d'abord parce qu'elle n'est mise en œuvre que lorsque le majeur est hors d'état de manifester sa volonté. En conséquence, elle ne s'appliquera que si la maladie d'Alzheimer dont souffre la personne atteint un stade évolué ${ }^{57}$. Or, il serait opportun d'étendre la mesure à une assistance de la personne au début de la maladie.

Ensuite, le cercle familial susceptible d'être habilité est limité. Dans un premier temps, le conjoint avait été ignoré du dispositif. Il ne pouvait ni saisir le juge des tutelles ni être désigné pour exercer la mesure ce qui était très contestable ${ }^{58}$ alors que le partenaire d'un pacte civil de solidarité ou le concubin y étaient autorisés. Une telle situation pouvait, d'ailleurs, créer divers conflits

52 G. Raoul-Cormeil, $L$ 'habilitation familiale: une tutelle adoucie, en la forme et au fond, préc.; D. Noguero, Les conditions de mise en æuvre de l'habilitation familiale, D. 2016.1510.

53 B. Mallet-Bricout, La nouvelle «habilitation familiale», ou le millefeuille de la représentation des majeurs protégés, RTD civ. 2016.190.

54 En ce sens également, D. Noguero, Les conditions de mise en æeuvre de l'habilitation familiale, préc.

55 Art. 494-4 c.civ.

56 Fondation Médheric Alzheimer, lettre de l'Observatoire oct. 2014/33-34.

57 T. Verheyde, Le rôle du juge dans la mesure d'habilitation familiale: de la lumière à l'éclipse!, Dr. Fam. 2016, n 11, dossier 42.

58 G. Raoul-Cormeil, L'habilitation familiale: une tutelle adoucie, en la forme et au fond, préc. 
par exemple entre le conjoint et un enfant né d'un premier mariage désirant l'écarter, ou encore entre un enfant habilité à gérer le patrimoine et le conjoint commun en biens qui admettrait difficilement une telle immixtion.

Cette exclusion s'expliquait sans doute par l'existence des mécanismes traditionnels de représentation issus du droit des régimes matrimoniaux (article 219 code civil). Toutefois, ceux-ci sont plus limités alors que le nouveau dispositif d'habilitation familiale est plus étendu en s'appliquant notamment aux actes personnels.

La législateur a finalement corrigé cette erreur: le conjoint de la personne hors d'état de manifester sa volonté du dispositif peut être habilitée sauf si la communauté de vie a cessés9, au même titre que les ascendants, descendants, frères et sœurs, partenaire d'un pacte civil de solidarité ou concubin.

Il demeure cependant regrettable qu'à la différence des mesures judiciaires, les alliés ou les amis, ou «une personne entretenant avec le majeur des liens étroits et stables $\rangle^{60}$ ne puissent ni exercer la mesure ${ }^{61}$, ni s'adresser au procureur afin de saisir le juge des tutelles. Les collatéraux ordinaires, même proches du majeur vulnérable, sont donc exclus, sans doute en raison de l'absence de contrôle judiciaire de la gestion ${ }^{62}$.

L'intéressé lui-même n'a pas qualité pour saisir le juge des tutelles alors même que le législateur entend reconnaître une autonomie à la personne vulnérable. Certes il le considère «hors d'état» mais il est cependant entendu, auditionnable. Or, en droit des majeurs protégés une personne peut toujours être requérante à une mesure de protection judiciaire pour elle-même ${ }^{63}$. La maladie d'Alzheimer étant évolutive, il conviendrait d'envisager d'ouvrir au malade la faculté de saisine du juge durant certaines périodes tant pour déclencher la mesure qu'en cas de difficultés d'exécution.

Enfin, cette mesure est critiquée car les tentations des proches peuvent être grandes de détournement. Il semble nécessaire de mieux protéger les intérêts de la personne vulnérable. Cela d'autant plus que la personne habilitée peut accomplir les actes d'administration ou de disposition pendant une durée assez longue dix ans voire vingt ans. Les troubles liés à la maladie d'Alzheimer s'aggravant,

59 Loi du 18 novembre 2016 de ratification de l'ordonnance n 2015-1288 du 15 octobre 2015.

60 Art. 430, al. 1er c.c.

61 L. Mauger-Vielpeau, L'habilitation familiale: la saisine du juge des tutelles, Dr. fam. 2016/11, dossier 45 .

62 Clarifications et reculs du droit des personnes et de la famille - À propos de la loi n ${ }^{\circ} 2015-177$ du 16 février 2015, N. Peterka, JCP G, 2015.243.

63 V. Montourcy, Le regard critique de l'avocat, AJ fam. 2015.605. 
il n'est guère envisageable en l'état actuel des connaissances médicales de lever la mesure plus tôt. La protection de la personne malade peut se révéler fragile car il n'est pas prévu de contrôle de gestion a posteriori ${ }^{64}$. Certes, si les intérêts du majeur protégé sont gravement menacés, le juge peut prendre les mesures nécessaires mais est-ce réellement suffisant? D'aucuns craignent que les libertés personnelles ne soient pas suffisamment garanties ${ }^{65}$.

Pour conclure, le mandat de protection future et l'habilitation familiale, mécanismes dont la primauté sur les mesures de protection judiciaire classiques est affirmée par le législateur, constituent une avancée dans la protection des majeurs. Des améliorations mériteraient d'y être apportées et notamment, à l'image des mesures judiciaires, une gradation de la protection.

\section{L'ineffectivité du mécanisme de gradation des mesures classiques de protection judiciaires}

Les différentes mesures judiciaires classiques sont subsidiaires non seulement par rapport aux mécanismes du droit commun de la représentation, des régimes matrimoniaux ou du mandat de protection future, mais également par rapport à une autre mesure de protection judiciaire moins contraignante. Elles sont certes plus lourdes mais permettent de moduler la protection en fonction de l'évolution de la maladie d'Alzheimer. Or, il apparaît que la mesure de sauvegarde de justice est insuffisamment utilisée comme mesure autonome (A) au profit des mesures de curatelle et surtout de tutelle.

\section{A. L'utilisation insuffisante de la sauvegarde de justice comme mesure autonome}

La sauvegarde justice au premier stade de la maladie. La mesure de protection doit être proportionnée et individualisée en fonction du degré d'altération des facultés personnelles de l'intéressé ${ }^{66}$ de sorte que la tutelle est subsidiaire par rapport à la curatelle, qui l'est elle-même par rapport à la sauvegarde de justice.

Cette gradation s'avère nécessaire concernant la maladie d'Alzheimer. La protection doit, néanmoins, toujours être assurée dans le respect des libertés individuelles, des droits fondamentaux et de la dignité de la personne. Le juge

${ }^{64}$ A. Batteur, Habilitation familiale et protection de la personne du majeur protégé, Etude, Dr. fam. 2016/11, dossier 45.

65 I. Maria, L'habilitation familiale à l'épreuve des principes directeurs du droit des majeurs protégés, Dr. fam. 2016/11, dossier 40.

66 Art. 428 c.c. 
des tutelles peut adapter la mesure en fonction de la progression de la maladie déclarée en envisageant dans un premier temps une mesure de surveillance par la sauvegarde de justice au premier stade de la maladie, par exemple lorsqu'on constate une multiplication des dépenses inconsidérées par l'utilisation démesurée de la carte bancaire et du chéquier.

Cette mesure, d'une durée maximale d'un an renouvelable une fois, concerne une personne ayant besoin d'une protection juridique temporaire ou d'être représentée pour l'accomplissement de certains actes déterminés ${ }^{67}$. Elle permet de la protéger sans la priver de sa capacité. La personne sous sauvegarde conserve l'exercice de ses droits mais les actes qu'elle a passés peuvent être remis en cause facilement par leur annulation s'ils la lèsent manifestement ou par leur réduction. Ce sera par exemple le cas lorsque la personne atteint de la maladie vend un terrain à un prix dérisoire ou a acheté des objets à un prix hors de proportion par rapport à leur valeur réelle ${ }^{68}$. Il est également possible, en application des textes de droit commun, de demander la nullité d'un acte pour trouble mental ${ }^{69}$.

La protection peut aussi être renforcée parce qu'il arrive que dans les instants de lucidité, des tiers mal intentionnés profitent de la faiblesse de la personne pour lui soutirer des sommes importantes. Le juge des tutelles peut dans cette hypothèse désigner un mandataire spécial en même temps que la mise sous sauvegarde de justice ou ultérieurement lorsque les troubles cognitifs s'aggravent. Celuici sera chargé d'accomplir un ou plusieurs actes d'administration et même de disposition rendus nécessaires par la gestion du patrimoine du majeur protégé qui ne pourra donc plus les accomplir. Il peut se voir également confier une mission de protection de la personne. Il pourra par exemple donner son accord pour des soins, la maintenir en milieu hospitalier si son état l'impose. La personne protégée elle-même a pu prendre la précaution de désigner un tel mandataire.

Le mandat de droit commun, telle une procuration bancaire, mis en place antérieurement à la mesure par la personne elle-même afin d'administrer ses biens n'est pas automatiquement remis cause mais le juge a le pouvoir de le révoquer ou de le suspendre après avoir entendu ou appelé le mandataire ${ }^{70}$. En pratique, pour éviter toute difficulté, la décision de mandat spécial prévoit quasi-systématiquement la révocation des procurations bancaires délivrées antérieurement ${ }^{71}$.
Art. 433 c.c.
V. art. 435 c.c.
Art. 414-1 c.c.
Art. 436, al. $1^{\text {er }}$ c.c.

71 F. Sauvage, Répertoire de procédure civile, préc. 
La restauration nécessaire de l'autonomie de la mesure. En pratique, la sauvegarde de justice est peu utilisée comme mesure autonome. Il est regrettable qu'elle soit essentiellement prononcée par le juge saisi d'une procédure de curatelle mais surtout de tutelle pour la durée de l'instance. Les principes de nécessité et de subsidiarité ne semblent donc pas suffisamment respectés.

L'autonomie de la mesure de sauvegarde de justice doit être restaurée. Elle peut constituer une première étape avant sa transformation d'abord en curatelle lorsque les troubles s'aggravent puis en tutelle. Cette maladie génère cependant des difficultés que les mesures de protection ne permettent pas toujours de résoudre. En outre, la modification de la mesure suppose une procédure assez lourde: le juge doit être saisi par un requérant et un certificat médical circonstancié tarifé doit être produit.

La plupart des majeurs sont finalement directement placés sous le régime de la curatelle et surtout de la tutelle.

\section{B. La curatelle et la tutelle}

La curatelle puis la tutelle ne devraient être prononcées qu'en fonction du degré d'altération des facultés mentales. Au fil du temps, les troubles cognitifs de la personne s'accentuent de sorte que la mesure de sauvegarde de justice ne peut plus assurer une protection suffisante. Une mesure d'assistance pourra alors être ordonnée avant la mise sous tutelle qui conduit à une représentation de la personne malade (1). Des principes communs gouvernent ces deux mesures (2).

\section{De l'assistance à la représentation}

La curatelle est une mesure d'assistance. Elle concerne la personne qui, sans être hors d'état d'agir elle-même, a besoin d'être assistée ou contrôlée d'une manière continue dans les actes importants de la vie civile. Celle-ci conserve le pouvoir de faire elle-même, et seule, les actes strictement personnels et les actes d'administration (gérer les revenus courants, payer les loyers, souscrire une assurance, conclure un bail d'une durée inférieure à neuf ans, ...). En revanche, les actes de disposition nécessitent l'assistance du curateur qui se manifeste par l'apposition de sa signature dans l'acte à côté de celle de la personne sous curatelle $^{72}$ (vendre un bien immobilier, faire une donation, en acquérir un, souscrire un crédit, une assurance-vie,...). Le curateur apporte également son aide au quotidien et a un devoir d'information.

72 Art. 467, al. 2 c.c. 
La curatelle apparait, dans un second temps, adaptée au patient atteint de la maladie d'Alzheimer parce que les troubles ponctuels dont il souffre par période, peuvent le conduire à effectuer des actes plus ou moins graves. C'est une mesure qui peut aussi être modulée en fonction de l'évolution de la maladie. En effet, elle peut être aménagée ${ }^{73}$ en fonction des facultés de compréhension et de gestion de la personne. Le juge détermine les actes que le majeur peut continuer à effectuer seul tels que le paiement de certaines factures, les dépenses d'un certain montant et les actes pour lesquels l'assistance du curateur est requise. Le plus souvent, cet aménagement est demandé concernant la conservation du chéquier par le majeur ${ }^{74}$ afin d'éviter des dépenses inconsidérées dont il ne se souviendrait pas.

La curatelle peut être également renforcée selon le degré d'altération des facultés de la personne s'il est prouvé que la personne a contracté des dettes injustifiées par rapport à ses revenus ou qu'elle présente des signes de confusion qui la mettent en danger. Dans ce cas, le curateur perçoit seul les revenus de la personne sur un compte ouvert au nom de cette dernière. Le juge peut aussi autoriser le curateur à conclure seul un bail d'habitation ou une convention d'hébergement afin d'assurer le logement de la personne protégée.

Lorsque la personne est en phase avancée de la maladie d'Alzheimer, les troubles mnésiques sont quasi-constants, la mesure de tutelle devient incontournable si aucune autre mesure n'a pu être mise en place. Elle constitue la mesure la plus complète et suppose que la personne a besoin d'être représentée d'une manière continue dans les actes importants de la vie civile. Le tuteur peut accomplir seul les actes d'administration. En revanche, il ne peut accomplir les actes de disposition qu'avec l'autorisation du juge des tutelles.

\section{Principes communs à la curatelle et à la tutelle}

Le majeur en curatelle ou en tutelle a pu lui-même désigné à l'avance son protecteur, sa volonté sera respectée sauf si son intérêt le commande. À défaut de désignation, le juge doit choisir par priorité une personne vivant avec le majeur (conjoint, partenaire pacsé ou concubin); et à défaut, un membre de la famille ou même un proche du majeur résidant avec lui ou entretenant avec lui des liens étroits et stables. Le juge tient compte des sentiments exprimés par la personne concernée par la mesure et de ses relations habituelles, des recommandations

\footnotetext{
Curatelle aménagée.

74 Pour un exemple, P. Bresson, Maladie d'Alzheimer et protection juridique, in: Protéger les personnes vulnérables, Regards croisés sur la profession de mandataire judiciaire à la protection des majeurs, F. Duriez, E. Luisin-Pagnod, M. Soulard-Pechberty, l'Harmattan 2014, p. 59 et $\mathrm{s}$.
} 
éventuelles des parents et proches. Si aucun d'eux ne «peut assumer» la mesure, le juge désigne un mandataire judiciaire à la protection des majeurs. Il peut aussi diviser l'exercice de la mesure en désignant un curateur ou un tuteur aux biens et un autre à la personne. En principe, cette mission est à la charge totale ou partielle du majeur protégé. Si ses revenus sont insuffisants, la collectivité publique l'assume.

Le curateur et le tuteur sont contrôlés par le juge des tutelles et le parquet. Un subrogé curateur ou subrogé-tuteur est désigné par le juge afin de surveiller la gestion, d'avertir le juge s'il constate des anomalies. Le juge des tutelles peut, par ailleurs, aménager la curatelle et la tutelle, tenir compte des souhaits du majeur et de sa capacité à effectuer certains actes.

Toute demande d'ouverture d'une mesure de protection suppose d'être accompagnée d'un certificat médical circonstancié rédigé par un médecin habilité. Afin d'assurer le respect des droits fondamentaux, le juge doit procéder à l'audition préalable du majeur à protéger. Il ne peut y passer outre que par une décision spécialement motivée et sur avis conforme du médecin habilité «si celle-ci est de nature à porter atteinte à sa santé ou s'il est hors d'état d'exprimer sa volonté» ${ }^{75}$. Cette dispense est donc exceptionnelle, le juge pouvant se déplacer sur le lieu de vie. L'audition doit lui permettre de mieux apprécier l'état et la situation personnelle du majeur. Or, en pratique, en raison de l'insuffisance des moyens confiés à la justice et de la réforme de la carte judiciaire, les dispenses d'audition sont très fréquentes ${ }^{76}$ ce qui est pour le moins inacceptable et dénoncé par le Défenseur des droits.

Concernant les décisions relatives à la personne, le principe est que le majeur décide seul dans la mesure où son état le permet. Cette autonomie est concevable lorsqu'il est au premier stade de la maladie d'Alzheimer et sous curatelle. Elle l'est moins lorsqu'il est à un stade avancé ayant justifié la mise sous tutelle. Si son état ne le permet pas, le juge peut décider que le curateur ou le tuteur l'assiste dans la prise de décision. Il peut donc décider d'inclure dans la mesure de protection, les décisions relatives à la personne en fonction de la capacité du majeur à comprendre et à exprimer sa volonté 77 . Ce n'est qu'à titre exceptionnel que tuteur ou le curateur seulement représente le majeur dans les décisions personnelles lorsqu'il est hors d'état de les prendre.

\footnotetext{
Art. 432 c.c.
}

76 Rapport du Défenseur des droits et Rapport de la Cour des Comptes, préc. Selon une étude réalisée par l'association nationale des juges d'instance, la dispense d'audition concernerait 60 à $80 \%$ des dossiers.

77 L. Pécaut-Rivolier, Protéger un majeur vulnérable, Delmas express 2012/1103, p. 205. 
En dehors de ce cas, si le majeur se met en danger, ils sont autorisés à prendre les mesures strictement nécessaires pour y mettre fin, situation qui peut se présenter s'agissant de la personne atteinte de la maladie d'Alzheimer.

Enfin, quant à la durée de ces deux mesures ${ }^{78}$, à leur ouverture, le juge des tutelles ne peut plus la fixer au-delà de cinq ans, ou dix ans pour la tutelle lorsque l'altération des facultés personnelles de l'intéressé n'apparait manifestement pas susceptible de connaître une amélioration selon les données acquises de la science. Lors du renouvellement ou de la révision de la mesure, le juge peut fixer une durée plus longue sans toutefois dépasser vingt ans ${ }^{79}$. Dans le cas du patient atteint de la maladie d'Alzheimer, la mesure ne peut donc pas durer plus de vingt ans. Le majeur n'aura cependant pas survécu.

Conclusion. Pour conclure, cette étude montre que la loi ne résout pas tous les problèmes soulevés par la complexité de la maladie d'Alzheimer. La législation actuelle offre une panoplie de mesures mais elles exigent des améliorations. Elles offrent une base à condition de respecter les principes fondamentaux qui les gouvernent et de fournir les moyens nécessaires afin d'assurer leur efficacité.

\section{References}

Aynès Laurent, Malaurie Philippe, Droit des personnes. La protection des mineurs et des majeurs, $8^{\mathrm{ème}}$ éd. par Ph. Malaurie, 2016, n 737, p. 345.

Batteur Annick, Habilitation familiale et protection de la personne du majeur protégé, Etude, Dr. fam. 2016/11, dossier 45.

Bévière-Boyer Bénédicte, Quel consentement pour les patients atteints par la maladie d'Alzheimer lors des recherches?, in: Consentement et santé, Dalloz, Thèmes et commentaires, p. 333 et s.

Bresson Patrick, Maladie d'Alzheimer et protection juridique, in: Protéger les personnes vulnérables, Regards croisés sur la profession de mandataire judiciaire à la protection des majeurs, F. Duriez, E. Luisin-Pagnod, M. Soulard-Pechberty, l'Harmattan 2014, p. 59 et s.

Caron-Déglise Anne, Protéger pour construire les capacités des personnes malades, in: Alzheimer, éthique et société, sous dir. de F. Gzil, E. Hirsch. ERES 2012, p. 303 et s.

Depadt-Sebag Valérie, L'EREMA à la recherche d'un statut des personnes atteintes de la maladie d'Alzheimer, D. 2011.2720.

Feuillet Brigitte, De la légitimité de la famille à l'intérêt du majeur protégé, in: Protéger les majeurs vulnérables. Quelle place pour les familles?, sous dir. de K. Leufeuvre, S. Moisdon-Chataigner, presses de l'EHESP, 2015, p. 23.

Fossier Thierry, L'objectif de la réforme du droit des incapacités, protéger sans jamais diminuer Defrénois 2005, n 38076, p. 3.

78 La loi $n^{\circ}$ 2015-177 du 16 février 2015 relative à la modernisation et à la simplification du droit.

79 Cette limite est selon la $\mathrm{CNCDH}$ une régression par rapport à la loi du 5 mars 2007. 
Gil Roger, Comment informer les patients atteints de troubles cognitifs?, RGDM 2016/59, p. 38 et s.

Glasson Christilla, Droit et personnes âgées: Mandat de protection future: quelles dispositions peut-on prendre pour ses vieux jours?, NPG Neurologie - Psychiatrie - Gériatrie 2011-11(62), p. 55.

Klein Julie, Le mandat de protection future ou la protection juridique conventionnelle, Dr. fam. 2007/21. Str.

Lefebvre-des-Noettes Véronique, Le point de vue d'un médecin: le choix de Sophie entre conflit de loyauté et conflit d'intérêt, in: Protéger les majeurs vulnérables. Quelle place pour les familles?, sous dir. de K. Leufeuvre, S. Moisdon-Chataigner, presses de 1'EHESP, 2015, p. 207 et s. spéc. p. 210.

Lefeuvre Karine, La place de la famille dans la loi du 5 mars 2007 portant réforme de la protection juridique des majeurs, in: Protéger les majeurs vulnérables. Quelle place pour les familles?, sous dir. de K. Leufeuvre, S. Moisdon-Chataigner, presses de l'EHESP, 2015, p. 31 et s.

Mallet-Bricout Blandine, La nouvelle «habilitation familiale», ou le millefeuille de la représentation des majeurs protégés, RTD civ. 2016.190.

Maria Ingrid, L'habilitation familiale à l'épreuve des principes directeurs du droit des majeurs protégés, Dr. fam. 2016/11, dossier 40.

Maria Ingrid, L'habilitation familiale, une nouvelle mesure de protection qui doit faire ses preuves, Dr. Fam. 2016/11, dossier 5.

Mauger-Vielpeau Laurence, L'habilitation familiale: la saisine du juge des tutelles, Dr. fam. 2016/11, dossier 45.

Montourcy Valéry, Le regard critique de l'avocat, AJ fam. 2015.605.

Noguéro David, Interrogations au sujet du mandat de protection future, Defrénois 2009, p. 1133.

Noguéro David, Les conditions de mise en œuvre de l'habilitation familiale, D. 2016, p. 1510.

Noguéro David, note Civ. $1^{\text {re }}, 12$ janv. 2011, D. 2011.1204.

Noguéro David, note sous Civ. ${ }^{\text {re }}, 4$ janv. 2017, D. 2017.191.

Pécaut-Rivolier Laurence, Protéger un majeur vulnérable, Delmas express, 2012, p. 44 et s.

Peterka Nathalie, Clarifications et reculs du droit des personnes et de la famille-À propos de la loi $n^{\circ}$ 2015-177 du 16 février 2015, JCP G, 2015.243.

Protéger les majeurs vulnérables. Quelle place pour les familles?, sous dir. de K. Leufeuvre, S. Moisdon-Chataigner, presses de l'EHESP, 2015, p. 193.

Raoul-Cormeil Gilles, Le mandat de protection future, un contrat pour préserver les biens professionnels ou l'intérêt de la famille, LPA 2014.

Raoul-Cormeil Gilles, Les personnes protégées et les dispositifs d'anticipation sur la fin de vie médicalisée, Dr. fam., oct. 2016/10, dossier 35.

Raoul-Cormeil Gilles, L'habilitation familiale: une tutelle adoucie, en la forme et au fond, D. 2015, 2335.

Rapport de la Cour des comptes, La protection juridique des majeurs: une réforme ambitieuse, une mise en œuvre défaillante, septembre 2016, http://www.ccomptes.fr/Accueil/Publications

Rapport du Défenseur des droits, Protection juridique des majeurs vulnérables, septembre 2016, http://www.defenseurdesdroits.fr/

Sauvage François, Répertoire de droit civil, Mandat de protection future, $\mathrm{n}^{\circ} 30$.

Sauvage François, Répertoire de procédure civile, préc. 
Semah Franck, Deberdt Walter, Ceccaldi Mathieu, De la maladie d'Alzheimer: apport de l'imagerie au florbétapir et autres radiopharmaceutiques de la plaque amyloïde, Médecine et droit, vol. 40 , p. 364 et s.

Verheyde Thierry, Capacité ou non du majeur à protéger à exprimer sa volonté: des incohérences, Dossier «Majeurs protégés: les nouveautés», AJ fam. 2016.236.

Verheyde Thierry, Le rôle du juge dans la mesure d'habilitation familiale: de la lumière à l'éclipse!, Dr. fam. 2016/11, dossier 42.

Verheyde Thierry, obs., AJ fam. 2011.110.

\section{Farida ARHAB-GIRARDIN}

\section{JAKIE SA ŚRODKI OCHRONNE DLA OSÓB Z CHOROBĄ ALZHEIMERA?}

(Streszczenie)

Liczba osób cierpiących na chorobę Alzheimera i inne choroby neurodegeneracyjne rośnie wraz ze starzeniem się populacji. W obecnym stanie wiedzy przyjmuje się, że zaburzenia zdolności umysłowych następują stopniowo i są nieodwracalne. Rodzi to wiele nowych pytań. Liczni bowiem pacjenci, mimo trudności poznawczych, wciąż mogą brać udział w podejmowaniu decyzji. Celem artykułu jest analiza adekwatności różnych istniejących środków służących ochronie osób dotkniętych tymi chorobami. Nowoczesne środki umownej lub sądowej ochrony wymagają udoskonalenia, a tradycyjne środki sądowe, mimo że nieskuteczne, mają z kolei tę zaletę, że z założenia pozwalają dostosować ochronę chorego do zmian jego stanu zdrowia.

Slowa kluczowe: pełnomocnictwo na przyszłość ds. spraw zdrowotnych; środki ochrony sądowej; umowne środki ochrony; choroba Alzheimera 